Running head: IMPLICIT SEXUALITY BIAS

\title{
Implicit preferences for straight people over lesbian women and gay men weakened from 2006 to 2013
}

\author{
Erin C. Westgate ${ }^{1}$ \\ Rachel G. Riskind ${ }^{2}$ \\ Brian A. Nosek ${ }^{1,3}$ \\ ${ }^{1}$ University of Virginia, Department of Psychology, \\ Box 400400, Charlottesville, VA, 22904, USA \\ ${ }^{2}$ Guilford College, Department of Psychology, \\ Greensboro, NC, 27410, USA \\ ${ }^{3}$ Center for Open Science, 210 Ridge McIntire Road, \\ Suite 500, Charlottesville, VA, 22911, USA
}

WORD COUNT: 5579

KEYWORDS: Implicit Association Test, culture, social psychology, implicit social cognition, sexual orientation

\section{Authors' Note:}

Correspondence can be sent to: Erin Westgate, University of Virginia, Department of Psychology, Box 400400, Charlottesville, VA 22904, USA. Email: ecw4za@virginia.edu. The authors of the paper contributed as follows: Designed research: B.N., E.W, R.R.; Analyzed data: E.W., R.R.; Wrote paper: E.W., R.R., B.N. We report all data exclusions, manipulations, and how we determined our sample sizes. We report all measures that were analyzed for this study. Additional measures are available in the dataset, and codebooks and data are available at: https://osf.io/p7va5/. Additional years of data are available here: https://osf.io/ctqxo/. This research was supported by Project Implicit.

Conflict of Interest Declaration: Nosek is an officer of Project Implicit Inc., a non-profit organization that includes in its mission "To develop and deliver methods for investigating and applying phenomena of implicit social cognition, including especially phenomena of implicit bias based on age, race, gender or other factors." 


\begin{abstract}
Legal rights and cultural attitudes towards lesbian women and gay men have shifted rapidly in the early $21^{\text {st }}$ century. Using 683,976 visitors to Project Implicit from February 2006 to August 2013, we investigated whether shifts were also observable in implicit evaluations that occur outside of conscious awareness or control. Similar to public opinion polling, the estimated explicit preference for straight people over lesbian women and gay men was $26 \%$ weaker on the last day compared to the first. The estimated implicit preference for straight people declined by $13.4 \%$ over the same period. The largest shifts in implicit evaluations occurred among Hispanic, White, female, liberal, and young adult participants; the smallest shifts occurred among Black, Asian, male, conservative, and older adult participants. Societal change in evaluation of lesbian and gay people is not limited to what people are willing and able to report. However, change in implicit evaluation appears to be slower.
\end{abstract}

KEYWORDS: Implicit Association Test, culture, social psychology, implicit social cognition, sexual orientation 
During the first part of the $21^{\text {st }}$ century, the legal rights and social roles of lesbian and gay people have been in flux. Positive and complex depictions of lesbian and gay television characters have become far more common in the U.S. (Kane, Gouttebroze, \& Townsend, 2013). Further, U.S. news media have extensively covered the suicides and peer rejection of LGBT adolescents as well as responses like Savage's It Gets Better campaign(Stetler, 2010). In 2010, the U.S. Congress and President Obama repealed the U.S. military's Don't Ask, Don't Tell policy, which had prohibited LGBT people from serving openly in the armed forces. Perhaps the most discussed issue was change in the legal recognition of same-sex couple relationships. In 2003, marriages between two people of the same gender were not legally recognized anywhere in the United States. In contrast, as of the end of 2014, 35 states, the District of Columbia, and the federal government legally recognize such marriages.

Accompanying the changes in legal rights, self-reported attitudes towards lesbian and gay people have also been shifting (Horn, 2012). National Gallup polls show that moral approval of lesbian and gay people in the U.S. has increased from $44 \%$ in 2006 to $59 \%$ in 2013 . In a worldwide poll, the Pew Global Attitudes Project found that in 2003 a slim majority of 51\% of respondents felt that homosexuality should be accepted by society. By 2013 , this number had increased to $60 \%$. In both cases, people who expressed positive attitudes towards lesbian and gay people were in the minority in the 2000s but constituted the majority of respondents by 2013 . Horn (2012) presented a multidimensional framework to describe predictors of self-reported attitudes. These include participant demographic (e.g., gender) and personality (e.g., gender role attitude) characteristics, as well as perceptions of the target and the type of self-report attitude measured. Despite change in self-reported attitudes, these predictors have remained relatively consistent across this time period (Horn, 2012). 
This shift in legal rights and cultural attitudes is dramatic. It prompts a question of significant theoretical interest in attitude research: what has changed? Self-reported attitudes can shift because people change their minds or because expressing one's mind becomes fraught with social consequences (Dasgupta \& Riviera, 2008; Lai, Hoffman, \& Nosek, 2013). It is possible that the shifts towards greater positivity toward lesbian women and gay men in past research are merely indicators of decreased willingness to express negative attitudes. In other words, are these changes in mental representation or have people just become more reluctant to admit to bias?

Implicit measures of evaluation offer an opportunity to test explanations for the observed change in attitudes. Should we attribute shifts in reported attitudes to shifts in evaluation? Or, are we observing shifts in willingness to express negative evaluations of lesbian and gay people - due to people's unwillingness to admit to or endorse those evaluations? Implicit measures assess evaluations without requiring an act of introspection and are relatively uncontrollable (Nosek, Hawkins, \& Frazier, 2011; 2012; and for discussion of interpretation of implicit and explicit attitude constructs see Banaji, 2001; Gawronski \& Bodenhausen, 2006; Nosek \& Hansen, 2008; Olson \& Fazio, 2004). If preferences for straight people compared to lesbian women and gay men show evidence of change on implicit measures as well as explicit measures, this would suggest that evaluations, not just reporting tendencies, are changing.

The present research used the Implicit Association Test (IAT) to investigate whether implicit attitudes towards lesbian and gay people have changed over time. The IAT is a computerized reaction time measure that assesses the comparative strength of associations between two pairs of concepts. Participants use two keys on a keyboard to categorize target stimuli appearing one at a time on the screen as belonging to one of two categories ("Gay people" or "Straight people") or one of two attributes ("Good" or "Bad"). If people are faster at 
accurately categorizing stimuli when "Gay people" and "Bad" are paired on the same side of the screen (compared to when "Straight people" and "Bad" are paired on the same side of the screen), it suggests that people have stronger negative associations with gay people than with straight people. The IAT thus can provide a measure of implicit evaluations of lesbian and gay people.

Moreover, there is considerable interest and evidence for the malleability of implicit evaluation in the short term (e.g., Lai, Marini, et al., 2014; Petty \& Brinol, 2010), but much less evidence for long-term or cultural shifts in evaluation (see Lai, Hoffman, \& Nosek, 2013 for a review). In order to determine the magnitude of change at the cultural level, researchers must examine shifts in mean bias across time. Knowing how and when implicit attitudes change on a cultural level is vitally important to understanding how long-term enduring changes in implicit attitudes occur. When large numbers of individuals experience small changes, these changes accumulate into large differences at the societal level. Thus, it is important to examine both change on the individual level (i.e., the association between date and an individual's bias score) as well as change in bias over time aggregated across many individuals (i.e., how much the sample mean changes over time).

To date, no researchers have identified any instances of implicit evaluation change on a cultural level. Such knowledge may help identify interventions and situations most likely to lead to lasting changes in implicit attitude evaluations. In the most similar study, Schmidt and Nosek (2010; $N=479,405)$ found little evidence for change in implicit racial preferences for Whites over Blacks between September 2006 and May 2009 that could have been attributed to the rise and election of Barack Obama as the first Black U.S. president. That lack of an effect was particularly interesting in light of evidence from laboratory research, which demonstrated that 
Obama could operate as a means of shifting implicit racial preferences short-term in the lab (Plant et al., 2009). Clearly, short-term malleability in the lab does not always translate to longterm social change. Thus, the present research may provide the first opportunity to demonstrate evidence of shifting implicit preferences across time on a cultural scale.

\section{Materials and Method}

Participants self-selected the "Sexuality IAT" from a list of available IATs after arriving on the Project Implicit demo site at https://implicit.harvard.edu and completing informed consent. The presentation order of the IAT, explicit measures, and demographics measures was randomized. After completing all measures, participants were debriefed with feedback on their IAT performance. All data were collected with human subjects approval from the University of Virginia.

Participants were volunteers who visited Project Implicit's demonstration site and selected the "Sexuality" Implicit Association Test (IAT) between February 6, 2006 and August 10, 2013, for a total of 7.48 years (2742 days) of data collection. February 6, 2006 marked the introduction of demographic items in the Sexuality IAT used in this analysis, and August 10, 2013 marked the initiation of data analysis for this project. We included only participants who had (a) complete data for all key measures and covariates and (b) an overall error rate of less than $30 \%$ or error rates below $40 \%$ on any individual block and a latency of $400 \mathrm{~ms}$ or less on fewer than 10\% of IAT trials (Greenwald, Nosek, \& Banaji, 2003; Nosek, Smyth et al., 2007). We excluded $3.6 \%$ of eligible sessions on the latter criterion. The final dataset consisted of 683,976 sessions, with a mean of $250(S D=215.23$; median $=214)$ sessions per day. For the final complete dataset, the smallest daily sample consisted of 1 session (during website downtime), and the largest consisted of 4664 sessions. The middle $80 \%$ of daily sample sizes 
ranged from 94 to 424 sessions. There was no evidence of systematic variation across time in the number of IAT sessions per day, $b=-.001, t(2729)=-.183, p=.86, R^{2}<.0001$.

Participants' average age was 24.9 years $(S D=10.6)$, and $62.3 \%$ of participants identified as female. Among participants, 70.8 percent reported their ethnicity as White (not of Hispanic origin), 6.4\% as Asian or Pacific Islander, 6.3\% as Black (not of Hispanic origin), 5.2\% multiracial (Other), 4.7\% as "other" or did not know, 4.6\% Hispanic, 1.5\% multi-racial (Black and White), and $0.6 \%$ as American Indian or Alaskan Native. Eighty percent of participants identified as heterosexual, $10.4 \%$ as lesbian, gay, or homosexual, and $9.6 \%$ as bisexual. Participants were relatively highly educated; $62.5 \%$ reported some college or an undergraduate college degree, $12.2 \%$ were completing or had completed an advanced degree, and $25.4 \%$ reported a high school education or less. Politically, 52\% of participants identified as liberal, $20.2 \%$ conservative, and $27.8 \%$ moderate. Most participants were U.S. residents $(83.2 \%)$.

Below, we report all measures that we analyzed for this study, and manipulations of order and procedural conditions for the IAT. Additional measures are available in the dataset. Complete codebooks and datafiles are available at: https://osf.io/p7va5/ and https://osf.io/ctqxo/

Explicit preferences for straight people compared to lesbian and gay people. Explicit attitudes for the first half of 2006 were assessed on a five-point Likert scale, ranging from -2 "I strongly prefer straight people to gay people" to +2 "I strongly prefer gay people to straight people." Explicit attitudes from the end of 2006 through 2013 were assessed on a seven-point Likert scale ranging from -3 "I strongly prefer straight people to gay people" to +3 "I strongly prefer gay people to straight people.” In both cases, the midpoint represented equal liking of 
straight and gay people. Scores from both scales were Z-transformed and combined into one standardized scale retaining the rational zero midpoint of no preference.

Implicit preferences for straight people compared to lesbian women or gay men. The IAT is a computerized reaction time measure that assesses the comparative strength of associations between two pairs of concepts (Greenwald, McGhee, \& Schwartz, 1998; Nosek, Greenwald, \& Banaji, 2007). Participants use two keys on a keyboard to categorize target stimuli appearing one at a time on the screen as belonging to one of four categories ("Gay people", "Straight people", "Good", "Bad"). Average response latencies in categorizing stimuli correctly are compared between two response conditions: (1) Gay people and Good items are categorized with one response key ('e') and Straight people and Bad items are categorized with the other ('i'); or, (2) Gay people and Bad items are categorized with one response key and Straight people and Good items are categorized with the other. Participants who categorize items faster in the first condition compared to the second are said to have an implicit preference for gay people compared to straight people.

The IAT followed the standard 7-block format described in Nosek, Greenwald, and Banaji (2005) and followed the same procedure as the sexuality IAT in Nosek, Smyth, et al. (2007) that reviewed its psychometric properties, variation across demographic groups, and relations with explicit measures. Order of the two category pairing conditions was randomized across participants, as was whether good items were categorized with either the 'e' or 'i' key (and bad items with the other key). Target stimuli for Straight people, Good, and Bad categories were the same for all participants. Target stimuli for Gay people consisted of pictures and words representing either lesbian women or gay men. The images consisted of 1) same-sex vs differentsex wedding toppers and 2) abstract restroom-style images of either two women, two men, or a 
woman and a man standing side-by-side. Participants were randomly assigned to see stimuli representing either lesbian women or gay men. This variation is known to shift the main effect such that participants show somewhat weaker implicit preferences for straight people over gay people when the Gay people category is represented with lesbian women compared to gay men (Nosek, Greenwald, \& Banaji, 2005, Supplement). We computed scores using the $D$ algorithm recommended by Greenwald et al. (2003) with response latencies recorded for the time used to make the correct response, even if an error occurred during the trial.

\section{Results}

We tested whether implicit evaluations of lesbian and gay people have changed over time, with 683,976 Project Implicit volunteers who participated between February 6, 2006 and August 10, 2013, for a total of 7.48 years (2742 days) of data collection. Overall, participants showed a preference for straight people over gay people both implicitly (IAT $D M=.33, S D=$ .47 , Cohen's $d=.70)$ and explicitly ${ }^{1}(M=.60, S D=1.26, d=.48)$. The implicit and explicit sexuality attitude measures were positively correlated, $r(654,328)=.437,95 \%$ CI $[.435, .439]$. We standardized the IAT and explicit scores, retaining the rational zero midpoint of no preference for the remainder of the analyses. $^{2}$

\section{Examining shifts in sample demographics over time}

\footnotetext{
${ }^{1}$ We report these descriptive statistics for explicit attitudes for the 7-point self-report measure in use between December 2006 and August 2013. Descriptive statistics for the 5-point self-report measure are available in the SI Appendix. Standardized scores for the 5- and 7-point scales are used in all subsequent analyses, covering the entire period of data collection.

${ }^{2}$ IAT scores were standardized independently for a few variations of the task procedure that were presented during the time period. Details of the original means and standardizing procedure are available in the SI Appendix.
} 
Inferring change with cross-sectional data is difficult. A particular inferential limitation would occur if the composition of the sample changed over time and that composition could account for observed shifts in evaluation. We examined shifts in demographics by regressing age, ethnicity, education, sexual orientation, political ideology, gender, and US residency on date of participation. There were slight shifts in demographic representation over the course of data collection (Total $\left.\mathrm{R}^{2}=.015\right)$. The largest shifts were for age and education: the sample became slightly younger and more educated over time ${ }^{3}$. To a lesser extent, the sample became slightly more female, White, conservative, less straight, and more composed of U.S. residents.

To account for potential influences, the hierarchical regression models ${ }^{4}$ evaluating the effects of date on implicit and explicit preferences first examined the effect of date in isolation, then tested whether the effect survived after adding these demographics to the model as covariates. Finally, we tested whether the effects of date on preferences were moderated by any of the demographic variables. In sum, when date effects on preferences were evaluated within demographic groups, the shift observed in the aggregate analysis persisted, suggesting that the small shifts in demographics over time could not account for the effects of date on implicit or explicit preferences reported next.

\section{Hierarchical regression evaluating shift in implicit preferences across time}

Though our primary research question is about societal change over time (i.e., sample mean change over time), we first examined variation in implicit preferences at the individual level. Many factors predict variation in attitudes across individuals - sex, race, education,

\footnotetext{
${ }^{3}$ Details regarding this regression analysis are available in Table 1 of the SI Appendix.

${ }^{4}$ Auto-correlation in the data is a potential concern when analyzing change across many consecutive days in a single time period. We observed minimal auto-correlation in the present data. See SI for additional information and analyses.
} 
political orientation, religiosity, and one's own sexual orientation - so variance accounted for by date of participation should be minor in comparison to these other sources of variation. Nevertheless, any indication of change at the individual level could accumulate to sizable evidence of change at the societal level.

We regressed IAT score on participation date to determine whether the magnitude of implicit sexuality preferences changed over time from February 2006 to August 2013 (see Table 1). Date was negatively related with implicit preference for straight people compared to gay people suggesting that the effect has weakened over time. Date accounted for roughly $0.1 \%$ of the variance in implicit preferences across individuals $\left(\mathrm{R}^{2}=.0009\right)$. In the second step, we added procedural variables for the implicit measure to the model: order of stimuli, gay men vs. lesbian women stimuli, and whether good items were categorized to the right or left. These variables accounted for about $4.2 \%$ of the variation in implicit evaluations but did not affect the relationship between date and implicit evaluation. 


\begin{tabular}{|c|c|c|c|c|c|}
\hline Predictor & $d f$ & $\eta_{p}^{2}$ & $F$ & $p$ & $R^{2}$ \\
\hline Step 1 & & & & & 0.0009 \\
\hline Date & 1 & 0.00087 & 593.92 & $<.0001$ & \\
\hline Step 2 & & & & & 0.0415 \\
\hline Date & 1 & 0.00105 & 716.57 & $<.0001$ & \\
\hline Side & 1 & 0.00025 & 173.95 & $<.0001$ & \\
\hline Order & 1 & 0.01975 & 13778.68 & $<.0001$ & \\
\hline Stimuli & 1 & 0.02144 & 14982.77 & $<.0001$ & \\
\hline Step 3 & & & & & 0.2677 \\
\hline Date & 1 & 0.00088 & 601.62 & $<.0001$ & \\
\hline Side & 1 & 0.00040 & 271.06 & $<.0001$ & \\
\hline Order & 1 & 0.02541 & 17835.04 & $<.0001$ & \\
\hline Stimuli & 1 & 0.02748 & 19328.49 & $<.0001$ & \\
\hline Age & 1 & 0.00165 & 1127.91 & $<.0001$ & \\
\hline Gender & 1 & 0.02240 & 15673.50 & $<.0001$ & \\
\hline Race & 7 & 0.01242 & 1228.85 & $<.0001$ & \\
\hline Sexual orientation & 2 & 0.13337 & 52629.40 & $<.0001$ & \\
\hline Political orientation & 1 & 0.04846 & 34833.17 & $<.0001$ & \\
\hline Education & 4 & 0.00459 & 788.44 & $<.0001$ & \\
\hline US Resident & 1 & 0.00037 & 251.44 & $<.0001$ & \\
\hline Step 4 & & & & & 0.2679 \\
\hline Date & 1 & 0.00088 & 10.02 & 0.0016 & \\
\hline Side & 1 & 0.00040 & 281.27 & $<.0001$ & \\
\hline Order & 1 & 0.02542 & 17838.72 & $<.0001$ & \\
\hline Stimuli & 1 & 0.02749 & 19335.74 & $<.0001$ & \\
\hline Age & 1 & 0.00163 & 1118.38 & $<.0001$ & \\
\hline Gender & 1 & 0.02244 & 15706.59 & $<.0001$ & \\
\hline Race & 7 & 0.01240 & 1208.27 & $<.0001$ & \\
\hline Sexual orientation & 2 & 0.13325 & 52533.89 & $<.0001$ & \\
\hline Political orientation & 1 & 0.04849 & 34880.86 & $<.0001$ & \\
\hline Education & 4 & 0.00457 & 783.65 & $<.0001$ & \\
\hline US Resident & 1 & 0.00038 & 257.54 & $<.0001$ & \\
\hline Date $\mathrm{x}$ Side & 1 & 0.00004 & 25.65 & $<.0001$ & \\
\hline Date $\mathrm{x}$ Order & 1 & 0 & 0.00 & 0.9534 & \\
\hline Date x Stimuli & 1 & 0.00002 & 10.81 & 0.0010 & \\
\hline Date $\mathrm{x}$ Age & 1 & 0.00007 & 45.21 & $<.0001$ & \\
\hline Date x Gender & 1 & 0.00001 & 4.67 & 0.0307 & \\
\hline Date x Race & 7 & 0.00006 & 5.79 & $<.0001$ & \\
\hline Date $\mathrm{x}$ Sexual orientation & 2 & 0.00002 & 5.79 & 0.0031 & \\
\hline Date $\mathrm{x}$ Political orientation & 1 & 0.00004 & 24.47 & $<.0001$ & \\
\hline Date $\mathrm{x}$ Education & 4 & 0.00001 & 1.63 & 0.1624 & \\
\hline Date x US Resident & 1 & 0 & 0.42 & 0.5146 & \\
\hline
\end{tabular}


Table 1. Hierarchical linear regression $(N=683,789)$ predicting implicit preference for straight people compared to lesbian or gay people by date (Step 1), IAT procedural variables (added in Step 2), demographic variables (added in Step 3), and their interactions (added in Step 4).

In the third step, we added demographic variables to the model: sexual orientation, political ideology, gender, race/ethnicity, age, education, and U.S. residency. As expected, these variables substantially increased the model's prediction of variation in implicit preferences to $26.8 \%$. In other words, individual differences in identity account for much more variation in implicit preferences than the date they completed the measure. Participants' sexual orientation and political ideology were particularly predictive. However, including demographic characteristics did not substantially reduce the effect of date predicting implicit preferences.

Finally, in the fourth step, we added tests of moderation: date (mean centered) interacting with each of the procedural and demographics variables entered in steps 2 and 3 . These interactions added very little additional prediction of implicit preferences to the model (change in model $\left.\mathrm{R}^{2}=.0002\right)$, and did not qualify date predicting implicit preferences.

In sum, we observed a very small effect of date on implicit preferences that survived the inclusion of many covariates and moderating terms. It is not surprising that at the individual level, demographic variables are far superior predictors of variation in implicit preferences than which date the participant completed the implicit measure. Substantial individual differences in implicit preferences persist over the timespan across sexual orientation, political ideology, and other variables. On an individual basis, time is a trivial predictor of implicit preferences compared to those identities. While the gaps between gay and straight people and liberals and conservatives may persist across time, the small date effect suggests that the entire culture may 
be shifting toward more positive evaluations of gay men and lesbians. In other words, if everyone develops more positive attitudes toward gay men and lesbians, then the variation across individuals by demographic factors will be preserved, making date a small contributor in accounting for variation across individuals, while still demonstrating a substantial societal shift.

Estimating the magnitude of change at the cultural level requires examining how the mean is shifting across time. Many small effects at the level of the individual may add up to large cumulative effects at the cultural level. To examine cultural change, we computed estimated effect sizes for the sample as a whole on the first and final days of data collection (see Table 3). For these estimates, we predicted standardized IAT $D$-scores for Day 1 and Day 2742 of data collection, calculated from the hierarchical regression analyses presented here. The effect size for date corresponds to an overall decline in the estimated effect size of $d=.77$ on Day 1 of the data collection to a $d=.67$ on Day 2742 (point estimate delta $=-.103$ ). Descriptive data for the mean standardized IAT $D$-scores for each month from 2006 to 2013 can be seen in Figure 1. In a separate analysis (see SI Appendix), we also tested for non-linear effects of time and found support for a quadratic effect in addition to the linear effect $\left(\mathrm{R}^{2}=.0013\right)$ suggesting that the rate of change accelerated slightly over the time period. In sum, across the nearly 7.5 years of data collection, the mean implicit preference for straight people over gay people declined by $13.4 \%$, a notable decline.

\section{Hierarchical regression evaluating shift in explicit preferences across time}

We used the same analysis plan to evaluate shifts in explicit preferences across time, excluding the step adding IAT procedural variables to the model. In the first step, we regressed participation date on explicit preferences (see Table 2). Date was negatively related to explicit preferences for straight people compared to gay people suggesting that the effect has weakened 
over time. Date accounted for about $0.17 \%$ of the variance in explicit preferences across individuals $\left(\mathrm{R}^{2}=.0017\right)$.

\begin{tabular}{|c|c|c|c|c|c|}
\hline Predictor & $d f$ & $\eta_{p}^{2}$ & $F$ & $p$ & $R^{2}$ \\
\hline Step 1 & & & & & .0017 \\
\hline Date & 1 & 0.0017 & 1086.1 & $<.0001$ & \\
\hline Step 2 & & & & & .3251 \\
\hline Date & 1 & 0.00143 & 934.89 & $<.0001$ & \\
\hline Age & 1 & 0 & 6.87 & $<.0001$ & \\
\hline Gender & 1 & 0.06065 & 42245.98 & $<.0001$ & \\
\hline Race & 7 & 0.00888 & 837.78 & $<.0001$ & \\
\hline Sexual orientation & 2 & 0.15620 & 103850.00 & $<.0001$ & \\
\hline Political orientation & 1 & 0.10497 & 76738.04 & $<.0001$ & \\
\hline Education & 4 & 0.00040 & 65.21 & $<.0001$ & \\
\hline US Resident & 1 & 0.00001 & 6.75 & 0.1000 & \\
\hline Step 3 & & & & & .3261 \\
\hline Date & 1 & 0.00143 & 28.02 & $<.0001$ & \\
\hline Age & 1 & 0.00001 & 7.89 & 0.0050 & \\
\hline Gender & 1 & 0.06058 & 39209.61 & $<.0001$ & \\
\hline Race & 7 & 0.00891 & 733.31 & $<.0001$ & \\
\hline Sexual orientation & 2 & 0.15650 & 56799.35 & $<.0001$ & \\
\hline Political orientation & 1 & 0.10519 & 72035.93 & $<.0001$ & \\
\hline Education & 4 & 0.00041 & 75.25 & $<.0001$ & \\
\hline US Resident & 1 & 0.00001 & 19.56 & 0.1000 & \\
\hline Date $\mathrm{x}$ Age & 1 & 0 & 0.02 & 0.8842 & \\
\hline Date $x$ Gender & 1 & 0.00003 & 19.47 & $<.0001$ & \\
\hline Date $x$ Race & 7 & 0.00002 & 2.26 & 0.0267 & \\
\hline Date $\mathrm{x}$ Sexual orientation & 2 & 0.00067 & 219.77 & $<.0001$ & \\
\hline Date $\mathrm{x}$ Political orientation & 1 & 0.00016 & 105.02 & $<.0001$ & \\
\hline Date $\mathrm{x}$ Education & 4 & 0.00011 & 17.37 & $<.0001$ & \\
\hline Date $\mathrm{x}$ US Resident & 1 & 0.00008 & 50.47 & $<.0001$ & \\
\hline
\end{tabular}

Table 2. Hierarchical linear regression $(N=683,789)$ predicting explicit preference for straight people compared to lesbian or gay people by date (Step 1), demographic variables (added in Step 2), and their interactions (added in Step 3). 
In the second step, adding demographics to the model substantially increased the model's prediction of variation in explicit attitudes to $33 \%$. As with implicit attitudes, participants' sexual orientation and political ideology accounted for the most variation. Even so, the effect of date predicting explicit preferences persisted. In the third step, adding interactions between date and the demographics added little additional prediction of explicit preferences to the model. Furthermore, these variables did not qualify the effect of date predicting explicit preferences.

As noted above, change at the cultural level is indicated by the mean change over time. We therefore computed regression estimated effect sizes for the sample as a whole on the first and final days of data collection. The effect size for date corresponds to a decline in estimated effect size from $d=.54$ on day 1 of the data collection to a $d=.40$ on day 2742 (Point estimate delta $=-.139$ ). In a separate analysis (see SI Appendix), we also tested for non-linear effects of time and found support for a quadratic effect in addition to a linear effect of time $\left(\mathrm{R}^{2}=.0030\right)$, suggesting that the rate of change accelerated slightly over the time period.

In sum, paralleling the results for implicit preferences, we found an effect of date on explicit attitudes that survived the inclusion of many covariates and moderating terms. All told, across the nearly 7.5 years of data collection, the mean explicit preference for straight people compared to gay people declined by $26 \%$ at the cultural level. This effect was approximately twice the size of the cultural shift observed using implicit measures.

\section{Demographic moderators of shifts in implicit preferences across time}

We conducted exploratory analyses to learn whether some demographic groups showed more evidence of shifting implicit preferences over time than others (i.e., the moderation effects in Step 4 of the hierarchical regression). Table 3 displays predicted standardized IAT $D$-scores for Day 1 and Day 2742 of data collection, calculated from the hierarchical regression analyses 
presented above ${ }^{5}$. Predicted scores for the first and last day were used in place of raw scores to represent the estimated trend in preferences across the time period. Positive point estimates represent pro-straight implicit preference, and negative point estimates represent pro-gay implicit preference, with a rational zero midpoint.

\footnotetext{
${ }^{5}$ In calculating estimates, we followed common practice in using the rational zero-point for politics, sample mode for categorical correlates, and sample median for continuous variables. Point estimates for Day 2742 are used in reporting group differences. All IAT procedural variables were entered as " 1 ", thus point estimates are for gay men stimuli, with incongruent pairings presented first, and "good" presented on the left side. Results remain consistent when IAT variables are entered as a " 2 " for lesbian women stimuli, congruent pairings presented first, and "good" presented on the right side.
} 

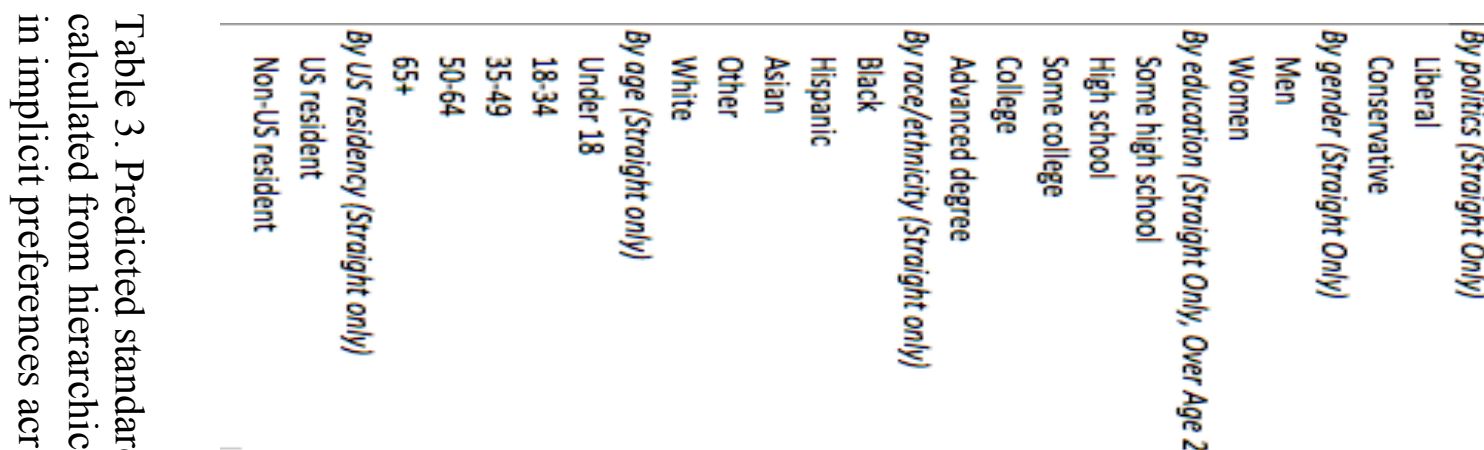

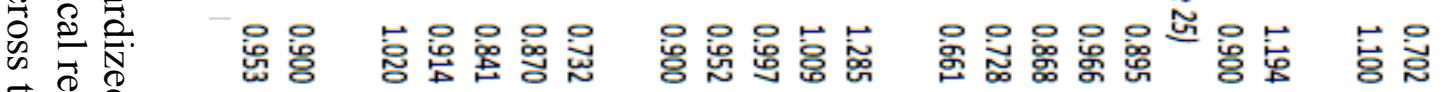

.

焉.

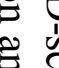

है

证

S

恶当

节.

هa

胥

$\rightarrow$

蛋怘

등. 웅

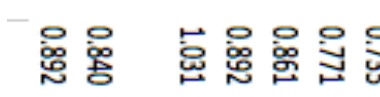

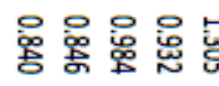

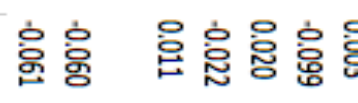

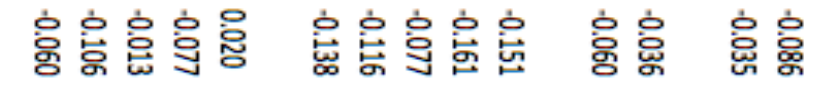

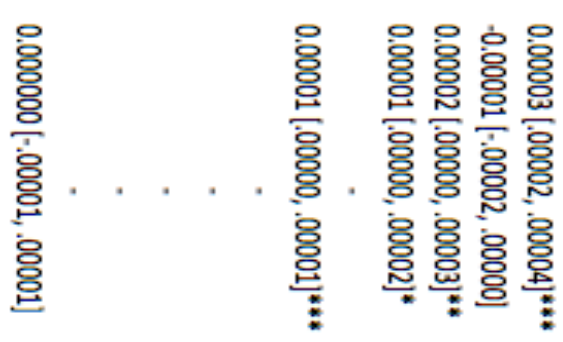

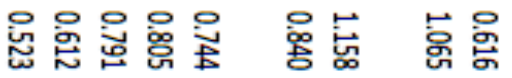

要

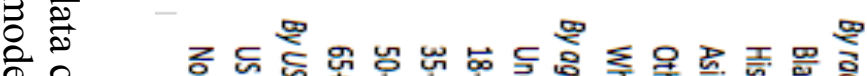
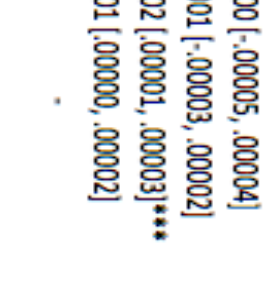

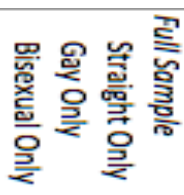

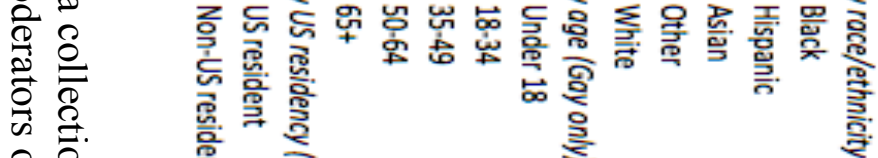

용

产

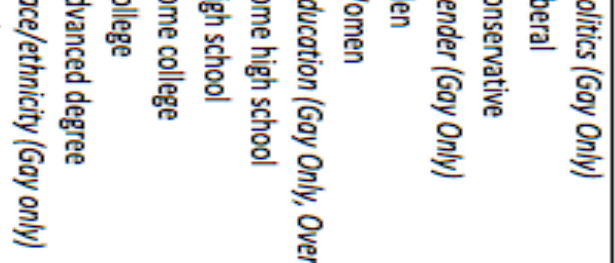

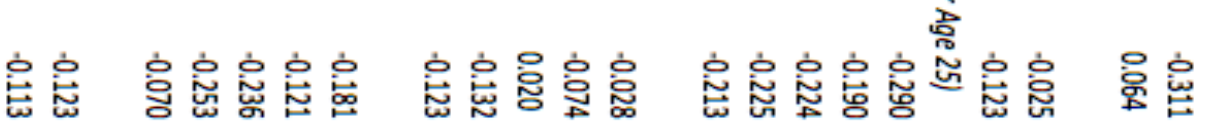

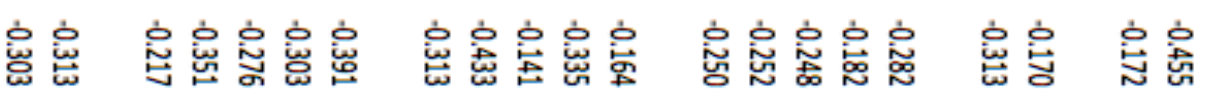

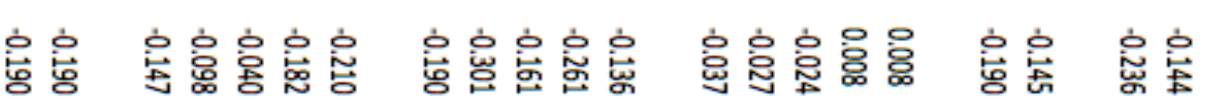
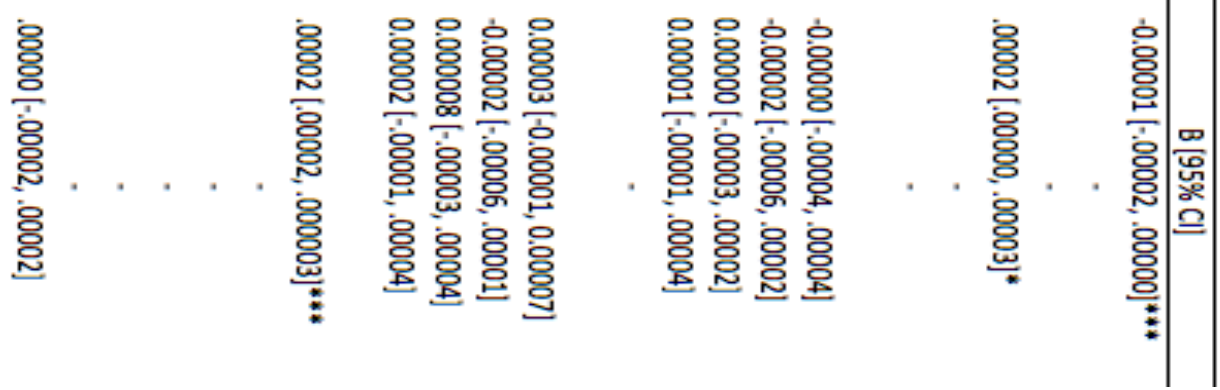

음형영

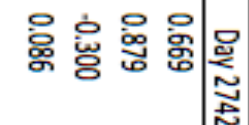

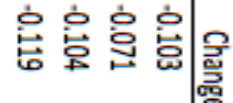

응 응응ㅇㅇㅇㅇㅇ

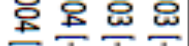

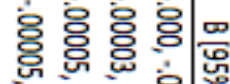

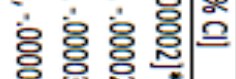

! 
Overall, race/ethnicity, age, political ideology were the strongest moderators. White and Hispanic people showed greater shifts than Asian and Black people, younger adults showed greater shifts than older adults, and liberals showed greater shifts than conservatives. Women showed slightly greater shifts in implicit preferences than men. Shifting implicit preferences were not confined to straight people; straight, bisexual, and lesbian or gay people all showed moderate shifts in implicit preferences during this time period, with bisexual people showing evidence of the largest shifts (see Figure 1). Overall, attitudes towards lesbian women shifted more than attitudes towards gay men. There was little evidence for differential change as a function of education or U.S. residency.

\section{$* * *$ INSERT FIGURE $1 * * *$}

Figure 1. Mean monthly implicit preference for straight people over gay people among straight, bisexual, and lesbian and gay participants from 2006 to 2013 with linear trendlines.

\section{Demographic moderators of shifts in explicit preferences across time}

We conducted similar exploratory analyses for explicit preferences (i.e., the moderation effects in Step 3 of the hierarchical regression). Overall, sexual orientation, political ideology, education, and U.S. residency were the largest moderators. Lesbian and gay participants reported weaker explicit preferences for gay people over straight people over time, whereas straight participants reported weaker explicit preferences for straight people over gay people over time. In other words, both groups reported weaker absolute preferences on the basis of sexual orientation over time. Conservatives shifted more than liberals, U.S. residents shifted more than non-U.S. residents, and less-educated people shifted more than highly educated people (college 
degree or higher). We observed a smaller effect for gender and race, with men shifting more than women and Black people shifting slightly more than White people. There was little evidence for differential change as a function of age.

\section{Discussion}

The cultural shift in legal rights and attitudes toward gay men and lesbians is not limited to what people are willing and able to report on explicit measures. In a very large sample collected between 2006 and 2013, we replicated evidence from public opinion polls for change over time in explicit attitudes towards lesbian and gay people. For the first time, we contributed evidence that this attitude shift has occurred implicitly, as well. This is the first documented evidence for a cultural shift in implicit evaluations about any topic. Implicit preference for straight people over gay people declined overall by 13.4\% from 2007 to 2013, and explicit preferences declined by $26 \%$ during that same period. Notably, the shift in explicit preferences was about twice the size of the shift in implicit preferences in this sample. This suggests that change is more rapid in evaluations that people report willingly than those that may operate outside of conscious awareness or conscious control.

Nearly all demographic groups showed weakening of implicit preferences for straight people over gay people over time, with the greatest changes occurring among Hispanic, White, female, liberal, and young adult participants. The smallest changes in implicit evaluations occurred among Black, Asian, male, conservative, and older adult participants. Interestingly, however, the relative magnitude of change was largely opposite for explicit preferences. Although nearly all groups reported weaker explicit preferences for straight people over gay people, shifts in explicit preferences were greatest among straight, male, conservative, Black, and less-educated participants. These are also the groups that held the strongest overall 
preferences for straight people. The two findings together offer a speculative interpretation: substantial explicit change may occur first and perhaps enable the slower implicit change that occurs later. Here, increases in explicit positivity toward gay men and lesbians may have already occurred among women and liberals prior to the assessed time period, setting the stage for later change in implicit preferences. Men and conservatives, however, may be currently undergoing explicit change, which could accelerate implicit change in the future. With the present data, however, this possibility is only speculation.

Evidence for the changing preferences of gay men and lesbian women furthers this speculation. Implicitly, these groups showed more substantial increases than straight participants in their positive evaluations of gay people compared to straight people. Explicitly, however, gay participants' reported preferences for gay compared to straight people weakened across the time period. That is, implicit and explicit changes were moving in opposite directions. It is as if explicit pro-gay preferences had become relatively extreme and were now becoming more moderate in the face of an increasingly tolerant culture, while implicit pro-gay preferences were continuing to strengthen, indicating the slow cumulative change over time. In the coming epoch, implicit preferences among lesbian women and gay men may likewise become more moderate, following the explicit trends. In sum, might explicit change be a leading indicator of eventual implicit change? Systematic investigation of this possibility is left to future research.

The current study has limitations. In particular, it is a cross-sectional comparison of a nonrepresentative sample of the U.S. and world populations who may be younger and less likely to identify themselves as male, conservative, or straight than the general population. Given that we do not have experimental control over the sampling, we cannot eliminate the possibility that an undetected selection effect is responsible for the apparent shift. However, this seems unlikely, as 
the effects are robust to controlling for the demographic variables available in the dataset, and our explicit data mirrors attitudinal shifts observed in Gallup and other representative polling. Also, the IAT stimulus set featured symbolic representations of gay and lesbian couples rather than individuals, and it may be difficult to compare relative change between the implicit and explicit measures due to variance in the measures' estimates. Finally, we were not able to examine religion as a potential moderator due to lack of data spanning the time period of interest. However, one strength is that we conducted daily assessments of implicit and explicit preferences towards gay and lesbian people, across more than seven years, with a massive sample, controlling for demographic characteristics. It is reasonable, but not definitive, to interpret the observed decline over time in preferences for straight people over gay people as indicating actual cultural change. Notably, despite the overall decline, participants still exhibited pronounced pro-straight preferences at the end of the time period. It would be useful to accumulate longitudinal evidence on representative samples in order to confirm this effect and provide parameter estimates for the magnitude of change across the culture.

A notable feature of this shift is that while we observed large shifts at the cultural level, the change in attitude for any single individual was trivial in effect size. The passage of time, and the cultural change occurring with it, accounts for an extremely small amount of a single person's implicit and explicit preferences, especially when compared to features of their identity such as sexual orientation and politics. What makes this observed change important is the fact that it is occurring across the entire sample, and across demographic categories, indicating a cultural shift in evaluation of lesbian and gay people. The tiny change for one individual accumulates into a substantial cultural effect expressed in all individuals $(13.4 \%$ decline for implicit preferences and 26\% decline for explicit preferences). For comparison, the effect size of 
time observed in this study was one hundred times greater than that found by Schmidt and colleagues (2010) when they examined change in implicit racial attitudes before and after President Obama's election. It is unclear whether a large change in a few people or a small change in everyone ultimately has a more substantial impact on society. We anticipate that most social change starts with large attitude changes within a subset of society and eventually ripples out to produce small changes in all members of society (Nosek, Banaji, \& Jost, 2009). In the early $21^{\text {st }}$ century, we appear to be bearing witness to this effect for evaluations of lesbian women and gay men. 


\section{References}

Banaji, M. R. (2001). Implicit attitudes can be measured. In H. L. Roediger III, J. S. Nairne, I. E. Neath, \& A. M. Surprenant (Eds.), The nature of remembering: Essays in honor of Robert G. Crowder (pp. 117-150). Washington, DC: American Psychological Association.

Dasgupta, N., \& Riviera, L. (2008). When social context matters: The influence of long-term contact and short-term exposure to admired out-group members on implicit attitudes and behavioral intentions. Social Cognition, 26, 112-123.

Gallup. (2013). "Regardless of whether or not you think it should be legal, for each one, please tell me whether you personally believe that in general it is morally acceptable or morally wrong. How about gay or lesbian relations?" May 2-7. [Survey report] in Gay and Lesbian Rights. Retrieved from http://www.gallup.com/poll/1651/gay-lesbianrights.aspx.

Gallup. (2006). "Regardless of whether or not you think it should be legal, for each one, please tell me whether you personally believe that in general it is morally acceptable or morally wrong. How about homosexual behavior?” May 8-11. [Survey report] in Gay and Lesbian Rights. Retrieved from http://www.gallup.com/poll/1651/gay-lesbianrights.aspx.

Gawronski, B., \& Bodenhausen, G. V. (2006). Associative and propositional processes in evaluation: an integrative review of implicit and explicit attitude change. Psychological Bulletin, 132, 692.

Greenwald, A. G., McGhee, D. E., \& Schwartz, J. L. (1998). Measuring individual differences in 
implicit cognition: the implicit association test. Journal of Personality and Social Psychology, 74, 1464-1480.

Greenwald, A. G., Nosek, B. A., \& Banaji, M. R. (2003). Understanding and using the implicit association test: I. An improved scoring algorithm. Journal of Personality and Social Psychology, 85, 197-216.

Horn, S.S. (2012). Attitudes about sexual orientation. In C.J. Patterson \& A. D’Augelli (Eds.). Handbook of Psychology and Sexual Orientation (pp. 239-251). New York: Oxford University Press.

Kane, M., Gouttebroze, M., \& Townsend, M. (2013). 2013 Network responsibility index. Retrieved from http://www.glaad.org/

Lai, C.K., Marini, M., Lehr, S.A., Cerrutl, C., Shin, J.E., Joy-Gaba, J.A. et al. (2014). Reducing implicit racial preferences: I. A comparative investigation of 17 interventions. Journal of Experimental Psychology: General.

Lai, C. K., Hoffman, K. M., \& Nosek, B. A. (2013). Reducing implicit prejudice. Social and Personality Psychology Compass, 7, 315-330.

Nosek, B. A., Banaji, M. R., \& Jost, J. T. (2009). The politics of intergroup attitudes. In J.T. Jost, A.C. Kay, \& H. Thorisdottir (Eds.). Social and Psychological Bases of Ideology and System Justification (pp. 480-506). New York: Oxford University Press.

Nosek, B. A., Greenwald, A. G., \& Banaji, M. R. (2005). Understanding and using the Implicit Association Test: II. Method variables and construct validity. Personality and Social Psychology Bulletin, 31, 166-180.

Nosek, B. A., Greenwald, A. G., \& Banaji, M. R. (2007). The Implicit Association Test at age 7: A methodological and conceptual review. In J.A. Bargh (Ed.). Social psychology and 
the Unconscious: The Automaticity of Higher Mental Processes, 265-292. Philadelphia: Psychology Press.

Nosek, B. A., \& Hansen, J. J. (2008). The associations in our heads belong to us: Searching for attitudes and knowledge in implicit evaluation. Cognition \& Emotion, 22, 553-594.

Nosek, B. A., Hawkins, C. B., \& Frazier, R. S. (2011). Implicit social cognition: From measures to mechanisms. Trends in Cognitive Sciences, 15, 152-159.

Nosek, B. A., Hawkins, C. B., \& Frazier, R. S. (2012). Implicit social cognition. In S. Fiske \& C.N. Macrae (Eds.), Handbook of Social Cognition, 31-53. New York: Sage.

Nosek, B. A., Smyth, F. L., Hansen, J. J., Devos, T., Lindner, N. M., Ranganath, K. A., ... \& Banaji, M. R. (2007). Pervasiveness and correlates of implicit attitudes and stereotypes. European Review of Social Psychology,18, 36-88.

Olson, M. A., \& Fazio, R. H. (2004). Reducing the influence of extrapersonal associations on the Implicit Association Test: personalizing the IAT. Journal of personality and social psychology, 86(5), 653.

Petty, R. E., \& Brinol, P. (2010). Attitude structure and change: Implications for implicit measures. In B. Gawronski \& B.K. Payne (Eds.), Handbook of implicit social cognition: Measurement, theory, and applications, 335-52. New York: Guilford Press.

Plant, E.A., Devine, P.G., Cox, W.T. L., Columb, C., Miller, S.L., Goplen, J., \& Peruche, B. M. (2009). The Obama effect: Decreasing implicit prejudice and stereotyping. Journal of Experimental Social Psychology, 45, 961-964.

Schmidt, K., \& Nosek, B. A. (2010). Implicit (and explicit) racial attitudes barely changed during Barack Obama’s presidential campaign and early presidency. Journal of Experimental Social Psychology, 46, 308-314. 
Stelter, B. (2010, October 18). Campaign offers to help gay youth. The New York Times. Retrieved from www.nytimes.com

\title{
Supporting Information (SI) for
}

Implicit preferences for straight people over lesbian women and gay men weakened from 2006 to 2013

\author{
Erin C. Westgate ${ }^{1}$, Rachel G. Riskind ${ }^{2}$, \& Brian A. Nosek ${ }^{1,3}$ \\ ${ }^{1}$ University of Virginia, ${ }^{2}$ Guilford College, ${ }^{3}$ Center for Open Science
}

This supplementary information provides additional details on the data and analyses reported in the paper. Additional measures are available in the dataset, and codebooks and data are available at: https://osf.io/p7va5/. Additional years of data are available here: https://osf.io/ctqxo/.

Results 


\section{Participant Exclusion on the Basis of IAT Error Rates}

We excluded from analyses any participants who had an overall error rate of over $30 \%$ on the IAT or error rates above $40 \%$ on any individual IAT block. Extreme error rates are generally indicative of participants who are not attempting to accurately categorize stimuli (i.e., random key presses). The error rate exclusions are based on the standard guidelines for scoring the IAT given in Greenwald, Nosek, \& Banaji (2003) and Nosek, Smyth et al. (2007). Greenwald et al. (2003) evaluated various criteria for respondent exclusion and the threshold and treatment (e.g., deletion vs replacement with the mean) of extremely slow or fast latencies, as well as trials with errors. Their recommendations focused on the scoring procedures that produced the strongest measurement validity, while retaining the greatest amount of data from both participants and individual trials

\section{Descriptives and Standardization of Explicit Attitudes}

Two versions of the self-report measure for explicit attitudes were used over the course of the study. Explicit attitudes for February 6, 2006 through December 6, 2006 were assessed on a five-point Likert scale, ranging from -2 "I strongly prefer straight people to gay people" to +2 "I strongly prefer gay people to straight people." Explicit attitudes from December 7, 2006 through 2013 were assessed on a seven-point Likert scale ranging from -3 "I strongly prefer straight people to gay people" to +3 "I strongly prefer gay people to straight people." In both cases, the midpoint represented equal liking of straight and gay people.

For both the 5-point $(M=.44, S D=.93, N=76,783)$ and 7-point measures $(M=.60, S D$ $=1.26, N=577,547$ ), participants reported a moderate pro-straight bias. For purposes of subsequent analysis, scores were Z-transformed for each scale and centered on a rational zero 
midpoint of no preference. The centered Z-scores were then combined into one standardized scale retaining the rational zero midpoint.

\section{Standardization of IAT Scores}

IAT scores were standardized independently for the eight different variations of the task procedure that were presented during the time period. Standardized scores were then combined into a single variable and centered on a rational zero point.

In version A of the IAT $(M=.31, S D=.46, N=94,996)$, gay men stimuli were used, with incongruent pairings presented first, and "good" presented on the left side. Version B $(M=$ $.44, S D=.43, N=94,630)$ used gay men stimuli, with congruent pairings presented first, and "good" presented on the left side. Version C $(M=.32, S D=.46, N=95,470)$ used lesbian women stimuli, with congruent pairings presented first, and "good" presented on the left side. Version D $(M=.22, S D=.49, N=95,638)$ used lesbian women stimuli, with incongruent pairings presented first, and "good" presented on the left side. Version $\mathrm{E}(M=.45, S D=.43, N=$ 76,278 ) used gay men stimuli, with congruent pairings presented first, and "good" presented on the right side. Version $\mathrm{F}(M=.34, S D=.46, N=75,745)$ used gay men stimuli, with incongruent pairings presented first, and "good" presented on the right side. Version G $(M=.22, S D=.49, N$ $=75,500$ ) used lesbian women stimuli, with incongruent pairings presented first, and "good" presented on the right side. Version $\mathrm{H}(M=.33, S D=.47, N=75,719)$ used lesbian women stimuli, with congruent pairings presented first, and "good" presented on the right side.

Standardized scores were first calculated separately for each of the 8 different versions. To compute standardized scores, that version's mean was subtracted from each raw score in the version and then divided by that version's standard deviation. The resulting standardized score 
was then centered at a rational zero point by subtracting the newly calculated standardized score that corresponded to a raw score of " 0 " for that version. This process was conducted separately for each version of the IAT, resulting in eight standardized scores retaining a rational zero point. These scores were then combined to create a composite standardized variable that retains a rational zero point representing no bias.

\section{Potential autocorrelation in IAT scores}

Auto-correlation in the data is a potential concern when analyzing change across many consecutive days in a single time period. Classic regression approaches (including the hierarchical regression analyses used in this paper) require that data meet the assumption of independent residuals, which is violated when the data are autocorrelated. To check that the data met the assumption of independent residuals, we examined the IAT data visually with a daily

plot of IAT means and conducted a Durbin-Watson test to determine the degree to which the data and residuals are autocorrelated. Generally, a Durbin-Watson test statistic close to 2 is preferred for linear regression. We found that there was little evidence of autocorrelation (Durbin-Watson test statistic $=1.97, \mathrm{p}<.05)$. This statistic, although significantly different from 2 (which would no indicate no autocorrelation in the data) due to the large sample size, falls within the generally accepted range of 1.5-2.5 required to meet assumptions for regression analysis.

\section{Test of non-linear effects of time on implicit attitudes}

In addition to the linear effect of time tested in the paper, we also tested for non-linear effects of time using hierarchical linear regression. In Step 1, we regressed a linear and squared term for participation date on IAT score to determine whether the addition of a non-linear term 
would increase the amount of variance accounted for in implicit sexuality preferences. Including the squared term resulted in better model fit $(\triangle \mathrm{AIC}=876)$, and increased the amount of variance accounted for by the model from $\mathrm{R}^{2}=.0009$ to $\mathrm{R}^{2}=.0013$. Both the linear and quadratic effects of time were negatively related to implicit preference for straight people compared to gay people, suggesting that implicit preferences for straight people have decreased over time and that this shift has accelerated over time.

In the second step, we added procedural variables for the implicit measure to the model order of stimuli, gay men vs. lesbian women stimuli, and whether good items were categorized to the right or left. These variables accounted for about $4.18 \%$ of the variation in implicit evaluations but did not affect the relationship between the linear and quadratic effects of date and implicit evaluation.

In the third step, we added demographic variables to the model: sexual orientation, political ideology, gender, race and ethnicity, age, education, and U.S. residency. Adding these substantially increased the model's prediction of variation in implicit preferences to $26.78 \%$. However, including demographics did not substantially reduce the quadratic or linear effects of date predicting implicit preferences.

Finally, in the fourth step, we added tests of moderation - the quadratic effect of date (mean centered) interacting with each of the procedural and demographics variables entered in steps 2 and 3. These interactions added very little additional prediction of implicit preferences to the model (change in model $\mathrm{R}^{2}=0$ ), but did not qualify the quadratic of effect of date predicting implicit preferences.

In sum, in addition to a linear effect of date, we also observed a very small quadratic effect of date on implicit preferences that survived the inclusion of many covariates and 
moderating terms, suggesting an acceleration in the decline in implicit preferences for straight people.

\section{Test of non-linear effects of time on explicit attitudes}

In addition to the linear effect of time tested in the paper, we also tested for non-linear effects of time on explicit attitudes using hierarchical linear regression. In Step 1, we regressed a linear and squared term for participation date on explicit attitudes to determine whether the addition of a non-linear term would increase the amount of variance accounted for in explicit sexuality preferences. Including the squared term resulted in better model fit and increased the amount of variance accounted for by the model from $\mathrm{R}^{2}=.0017$ to $\mathrm{R}^{2}=.003$. Both the linear and quadratic effects of time were negatively related to explicit preference for straight people compared to gay people, suggesting that explicit preferences for straight people have decreased over time and that this shift has accelerated over time.

In the second step, we added demographic variables to the model: sexual orientation, political ideology, gender, race and ethnicity, age, education, and U.S. residency. Adding these substantially increased the model's prediction of variation in explicit preferences to $32.59 \%$. However, including demographics did not substantially reduce the quadratic or linear effects of date predicting explicit preferences.

Finally, in the third step, we added tests of moderation - the quadratic effect of date (mean centered) interacting with each of the demographics variables entered in step 2. These interactions added very little additional prediction of explicit preferences to the model (change in model $\left.\mathrm{R}^{2}=0.0002\right)$, but did qualify the quadratic of effect of date predicting explicit preferences. 
Gender and sexual orientation emerged as the strongest moderators of the quadratic effect of time, followed by political ideology and U.S. residency.

In sum, in addition to a linear effect of date, we also observed a very small quadratic effect of date on explicit preferences that survived the inclusion of many covariates and was moderated by gender and sexual orientation, suggesting an acceleration in the decline in explicit preferences for straight people. 


\begin{tabular}{lccccc}
\hline \multicolumn{1}{c}{ Demographics } & $d f$ & $\eta_{\mathrm{p}}^{2}$ & $F$ & $p$ & $R^{2}$ \\
\hline Demographic Shifts & & & & & .0154 \\
Age & 1 & 0.00760 & 5236.20 & $<.0001$ & \\
Gender & 1 & 0.00090 & 613.87 & $<.0001$ & \\
Race & 7 & 0.00236 & 231.19 & $<.0001$ & \\
Sexual orientation & 2 & 0.00073 & 249.13 & $<.0001$ & $<.0001$ \\
Political orientation & 1 & 0.00012 & 81.30 & $<.0001$ & \\
Education & 4 & 0.00376 & 645.66 & $<.0001$ & \\
US Resident & 1 & 0.00066 & 450.94 & $<$ \\
\hline
\end{tabular}

Table S1. Shifts in demographics examined by regressing age, ethnicity, education, sexual orientation, political ideology, gender, and US residency on date of participation. 
Running head: IMPLICIT SEXUALITY BIAS 


\begin{tabular}{|c|c|c|c|c|c|}
\hline Predictor & $d f$ & $h_{p}^{2}$ & $F$ & $p$ & $R^{2}$ \\
\hline Step 1 & & & & & 0.0013 \\
\hline Date & 1 & 0.00090 & 617.37 & $<.0001$ & \\
\hline Date $^{2}$ & 1 & 0.00041 & 282.80 & $<.0001$ & \\
\hline Step 2 & & & & & 0.0418 \\
\hline Date & 1 & 0.00104 & 716.15 & $<.0001$ & \\
\hline Date $^{2}$ & 1 & 0.00033 & 228.12 & $<.0001$ & \\
\hline Side & 1 & 0.00016 & 107.29 & $<.0001$ & \\
\hline Order & 1 & 0.01975 & 13783.47 & $<.0001$ & \\
\hline Stimuli & 1 & 0.02145 & 14991.02 & $<.0001$ & \\
\hline Step 3 & & & & & 0.2678 \\
\hline Date & 1 & 0.00087 & 597.94 & $<.0001$ & \\
\hline Date $^{2}$ & 1 & 0.00015 & 103.37 & $<.0001$ & \\
\hline Side & 1 & 0.00031 & 208.77 & $<.0001$ & \\
\hline Order & 1 & 0.02542 & 17837.59 & $<.0001$ & \\
\hline Stimuli & 1 & 0.02750 & 19334.53 & $<.0001$ & \\
\hline Age & 1 & 0.00168 & 1149.87 & $<.0001$ & \\
\hline Gender & 1 & 0.02241 & 15680.25 & $<.0001$ & \\
\hline Race & 7 & 0.01242 & 1228.79 & $<.0001$ & \\
\hline Sexual orientation & 2 & 0.13327 & 52584.36 & $<.0001$ & \\
\hline Political orientation & 1 & 0.04846 & 34829.57 & $<.0001$ & \\
\hline Education & 4 & 0.00457 & 784.56 & $<.0001$ & \\
\hline US Resident & 1 & 0.00039 & 266.78 & $<.0001$ & \\
\hline Step 4 & & & & & 0.2678 \\
\hline Date & 1 & 0.00083 & 566.17 & $<.0001$ & \\
\hline Date $^{2}$ & 1 & 0.00015 & 7.36 & 0.0067 & \\
\hline Side & 1 & 0.00030 & 71.68 & $<.0001$ & \\
\hline Order & 1 & 0.02542 & 8001.19 & $<.0001$ & \\
\hline Stimuli & 1 & 0.02750 & 8254.14 & $<.0001$ & \\
\hline Age & 1 & 0.00170 & 427.39 & $<.0001$ & \\
\hline Gender & 1 & 0.02240 & 6894.10 & $<.0001$ & \\
\hline Race & 7 & 0.01242 & 537.53 & $<.0001$ & \\
\hline Sexual orientation & 2 & 0.13323 & 22315.69 & $<.0001$ & \\
\hline Political orientation & 1 & 0.04846 & 14473.65 & $<.0001$ & \\
\hline Education & 4 & 0.00457 & 316.75 & $<.0001$ & \\
\hline US Resident & 1 & 0.00039 & 87.30 & $<.0001$ & \\
\hline Date $^{2} \times$ Side & 1 & 0.00004 & 2.40 & 0.1212 & \\
\hline Date $^{2}$ x Order & 1 & 0.00001 & 10.18 & 0.0014 & \\
\hline Date $^{2}$ x Stimuli & 1 & 0 & 0.09 & 0.7632 & \\
\hline Date $^{2} \times$ Age & 1 & 0.00002 & 1.52 & 0.2179 & \\
\hline Date $^{2}$ x Gender & 1 & 0 & 3.17 & 0.0752 & \\
\hline
\end{tabular}




$\begin{array}{lllll}\text { Date }^{2} \times \text { Race } & 7 & 0.00003 & 2.54 & 0.0131 \\ \begin{array}{l}\text { Date } \\ \text { Date }\end{array} \text { x Sexual orientation } & 2 & 0.00002 & 6.51 & 0.0015 \\ \begin{array}{l}\text { orientation } \\ \text { Date }\end{array} & 1 & 0.00001 & 5.39 & 0.0203 \\ \text { Date }^{2} \times \text { Education } & 4 & 0.00001 & 2.20 & 0.0666 \\ \end{array}$

Table S2. Hierarchical linear regression $(N=683,789)$ predicting linear and quadratic effects of date on implicit preference for straight people compared to lesbian or gay people (Step 1), with additional predictors of IAT procedural variables (added in Step 2), demographic variables (added in Step 3), and their interactions (added in Step 4). 


\begin{tabular}{|c|c|c|c|c|c|}
\hline Predictor & $d f$ & $\mathrm{~h}_{\mathrm{p}}^{2}$ & $F$ & $p$ & $R^{2}$ \\
\hline Step 1 & & & & & 0.0030 \\
\hline Date & 1 & 0.0018 & 1149.98 & $<.0001$ & \\
\hline Date $^{2}$ & 1 & 0.0013 & 878.68 & $<.0001$ & \\
\hline Step 2 & & & & & 0.3259 \\
\hline Date & 1 & 0.00150 & 980.44 & $<.0001$ & \\
\hline Date $^{2}$ & 1 & 0.00118 & 770.05 & $<.0001$ & \\
\hline Age & 1 & 0.00002 & 12.34 & 0.0004 & \\
\hline Gender & 1 & 0.06077 & 42333.32 & $<.0001$ & \\
\hline Race & 7 & 0.00887 & 836.23 & $<.0001$ & \\
\hline Sexual orientation & 2 & 0.15608 & 60504.33 & $<.0001$ & \\
\hline Political orientation & 1 & 0.10506 & 76809.95 & $<.0001$ & \\
\hline Education & 4 & 0.00039 & 63.75 & $<.0001$ & \\
\hline US Resident & 1 & 0 & 1.41 & 0.2355 & \\
\hline Step 3 & & & & & 0.3261 \\
\hline Date & 1 & 0.00157 & 1029.03 & $<.0001$ & \\
\hline Date $^{2}$ & 1 & 0.00118 & 0.01 & 0.9038 & \\
\hline Age & 1 & 0.00002 & 0.87 & 0.3513 & \\
\hline Gender & 1 & 0.06082 & 19981.27 & $<.0001$ & \\
\hline Race & 7 & 0.00886 & 370.83 & $<.0001$ & \\
\hline Sexual orientation & 2 & 0.15610 & 26641.03 & $<.0001$ & \\
\hline Political orientation & 1 & 0.10513 & 34012.47 & $<.0001$ & \\
\hline Education & 4 & 0.00039 & 1.92 & $<.0001$ & \\
\hline US Resident & 1 & 0 & 8.02 & 0.0046 & \\
\hline Date $^{2} \times$ Age & 1 & 0 & 2.87 & 0.0902 & \\
\hline Date $^{2} \times$ Gender & 1 & 0.00014 & 94.35 & $<.0001$ & \\
\hline Date $^{2} \times$ Race & 7 & 0 & 0.70 & 0.6715 & \\
\hline Date $^{2} \mathrm{x}$ Sexual orientation & 2 & 0.00015 & 49.29 & $<.0001$ & \\
\hline Date $^{2} \times$ Political orientation & 1 & 0.00003 & 20.95 & $<.0001$ & \\
\hline Date $^{2} \mathrm{x}$ Education & 4 & 0.00001 & 1.92 & 0.1042 & \\
\hline Date $^{2} \times$ US Resident & 1 & 0.00003 & 19.22 & $<.0001$ & \\
\hline
\end{tabular}

Table S3. Hierarchical linear regression $(N=683,789)$ predicting explicit preference for straight people compared to lesbian or gay people by linear and quadratic effects of date (Step 1), demographic variables (added in Step 2), and their interactions (added in Step 3) 
Running head: IMPLICIT SEXUALITY BIAS 
